\title{
Исследование возможности
}

снижения массы бронепанелей путем применения комплексных трехслойных пластин из стальных сплавов в кабинах бронеавтомобилей

\author{
В.Н. Зузов, Н. Шаш \\ МГТУ им. Н.Э. Баумана, 105005, Москва, Российская Федерация, 2-я Бауманская ул., д. 5, стр. 1
}

\section{A Study of the Possibility of Reducing the Weight of Armored Plating Using Complex Three-Layer Alloy Steel Plates for Armored Vehicle Cabins}

\author{
V.N. Zuzov, N. Shash \\ BMSTU, 105005, Moscow, Russian Federation, $2^{\text {nd }}$ Baumanskaya St., Bldg. 5, Block 1 \\ e-mail: zuzvalery@rambler.ru,nebrasshash@mail.ru
}

Рассмотрен процесс пробития пулей 7,62 мм «АРМ2» с жестким стальным сердечником (являющейся одной из самых распространенных среди аналогов) комплексных трехслойных пластин из стального сплава Armox 560T, используемого для бронезащиты, суммарной толщиной до 10,5 мм. Исследования проведены для восьми моделей с разными геометрическими параметрами при скорости удара пули 830 м/с. Выполнены анализ и сравнение по массе, баллистике и обеспечению уровня защиты BR7 по европейскому стандарту EN 1063 между комплексными трехслойными пластинами и монослойной пластиной толщиной 12 мм, изготовленной из той же стали. Приведены разработанные рациональные конечно-элементные модели комплексных трехслойных пластин и пуль, с помощью которых получены результаты с приемлемой точностью и минимальным временем решения при использовании программного комплекса LS-DYNA. Основные результаты расчетов включают в себя значения остаточных скорости и кинетической энергии пули после пробития трехслойной пластины, а также определение ее лучших геометрических параметров, которые рекомендовано использовать в бронекабине автомобиля.

Ключевые слова: стальной сплав, пуля «АРМ2», комплексные трехслойные пластины, EN1063, LS-DYNA, конечно-элементные модели, бронекабина автомобиля

In this paper, the authors study the process of penetrating complex three-layer plates made of Armox $560 \mathrm{~T}$ alloy steel of the total thickness of $10.5 \mathrm{~mm}$ by a $7.62 \mathrm{~mm}$ ARM2 bullet with a hard steel core (one of the most commonly occurring types). The study is conducted on eight models with different geometrical parameters and the bullet velocity of $830 \mathrm{~m} / \mathrm{s}$. Complex three-layer plates and a monolayer plate of the $12 \mathrm{~mm}$ thickness made of the same type of steel are analyzed and compared by weight, ballistic characteristics and the level of protection BR 7 according to the European standard EN 1063. The developed rational finite element models are presented for the complex three-layer plates and bullets. The models allow obtaining results with an acceptable accuracy and the minimal solution time using the LS-DYNA software complex. The main calculation results include the residual velocity and kinetic 
energy of the bullet after penetrating the three-layer plate, as well as the determination of its best geometric parameters that are recommended for use in armored vehicle cabins.

Keywords: alloy steel, APM2 bullet, complex three-layer plates, EN 1063, LS-DYNA, finite element model, armored cabin

Оптимизация металлических щитов по разным параметрам и критериям против воздействия снарядов давно представляет практический интерес в военных и гражданских целях. Проведено множество экспериментальных, численных и теоретических исследований по оценке сопротивления прониканию снарядов в моно- и многослойные металлические пластины [1-5]. При этом основными требованиями к кабинам бронированных автомобилей являются малая масса и минимальная стоимость при обеспечении необходимого уровня бронезащиты. Выполнение этих требований одна из самых важных и непростых задач, с которыми сталкиваются создатели бронекабин.

Известно, что многослойные бронепанели (пластины) и оболочки (в том числе трехслойные) обладают как достоинствами, так и недостатками [6]. Трехслойные металлические пластины также используют для бронезащиты [7]. Главный плюс их применения - возможность снижения массы при обеспечении требуемой бронезащиты, а основные сдерживающие факторы - сложность изготовления и более высокая стоимость по сравнению с одно- и двухслойными конструкциями.
Для достижения эффективной защиты при минимальной массе конструкции необходимо задать определенные толщины всех пластин и углы наклона средней из них. В связи с этим исследовано пробитие комплексных трехслойных пластин (КТП) из стального сплава Armox 560T суммарной толщиной до 10,5 мм (это значение меньше минимальной толщины двухслойной пластины (12 мм) из того же материала, обеспечивающей непробитие пулей 7,62 мм «АРМ2» с жестким стальным сердечником (далее - пулей «АРМ2») [8]) для восьми разных моделей КТП при скорости удара пули 830 м/с. Также выполнены анализ и сравнение КТП с двухслойной пластиной толщиной 12 мм из той же стали, исследованной в работе [8], по массе, баллистике и соответствию уровню защиты BR7 по европейской норме EN 1063.

Цель работы - обоснование выбора толщин пластин и угла наклона средней из них в КТП, обеспечивающих уровень защиты BR7 по европейской норме EN 1063 и минимальную массу конструкции, а также оценка целесообразности их применения.

Численное моделирование. Изучен процесс пробития пулей «АРМ2» при скорости удара
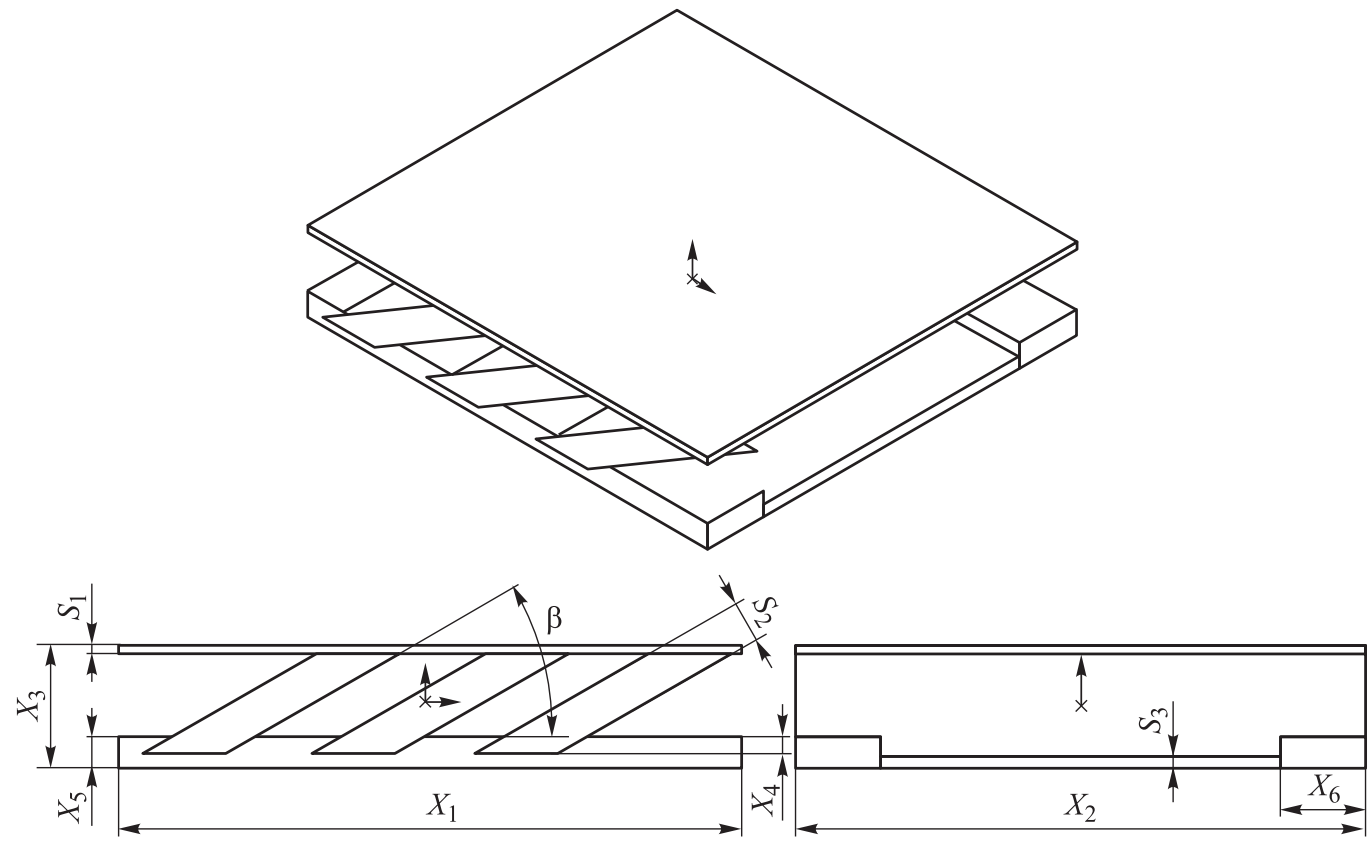

Рис. 1. Модель КТП 


\section{Размеры моделей}

\begin{tabular}{|c|c|c|c|c|c|c|c|c|}
\hline \multirow{2}{*}{ Номер модели } & $S_{1}$ & $S_{2}$ & $S_{3}$ & $X_{1}$ & $X_{3}$ & $X_{4}$ & $X_{5}$ & \multirow{2}{*}{$\beta$, град } \\
\hline & \multicolumn{7}{|c|}{ MM } & \\
\hline 1 & 3 & 3 & 3 & 70 & 20 & 2 & 5 & 35 \\
\hline 2 & 4 & 4 & 2 & 72 & 20 & 3 & 5 & 35 \\
\hline 3 & 3 & 5 & 2 & 74 & 19 & 3 & 5 & 35 \\
\hline 4 & 2 & 6 & 2 & 76 & 18 & 3 & 5 & 35 \\
\hline 5 & 1 & 7 & 2 & 95 & 17 & 3 & 5 & 30 \\
\hline 6 & 1 & 7 & 2 & 109 & 21 & 3 & 5 & 30 \\
\hline 7 & 1 & 7 & 2 & 78 & 17 & 3 & 5 & 35 \\
\hline 8 & 0,5 & 8 & 2 & 100 & 17 & 3 & 5 & 30 \\
\hline
\end{tabular}

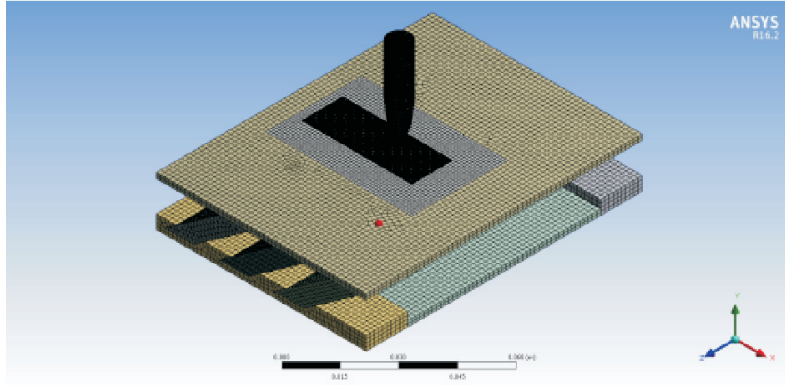

$a$

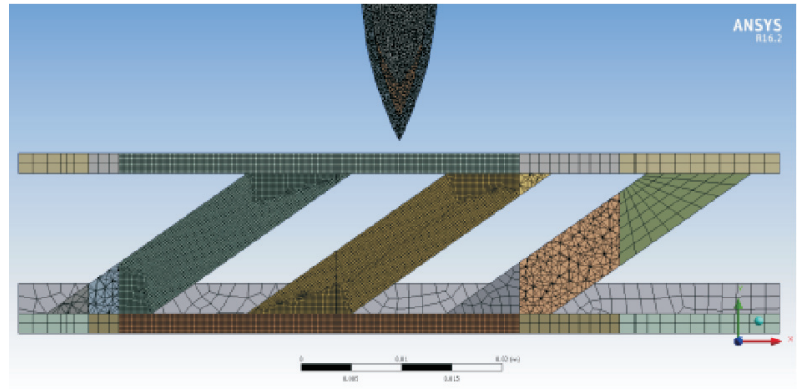

6

Puc. 2. Трехмерная КЭМ пули и пробиваемой пластины (a) и сечение в КЭМ (б), полученные для модели 4 (55Ar2-6-2 мм)

830 м/с КТП из стального сплава Armox 560T суммарной толщиной до 10,5 мм для восьми разных моделей, обозначенных как $\alpha A r S_{1}-S_{2}-S_{3}$ мм, где $\alpha-$ угол контакта между пулей и средней пластиной; Ar - сплав Armox 560T; $S_{1}, S_{2}$ и $S_{3}$ - толщина верхней, средней и нижней пластины соответственно. Для исследования использованы следующие восемь моделей: 1 - 55Ar3-3-3 мм; 2 - 55Ar4-4-2 мм, 3 - 55Ar3-5-2 мм; 4 - 55Ar2-6-2 мм; 5 60Ar1-7-2 мм; 6 - 60Ar1-7-2 мм мод; 7 55Ar1-7-2 мм; 8 - 60Ar0,5-8-2 мм. Угол наклона средней пластины $\beta$ равен 30 или $35^{\circ}$.

Средняя пластина задана самой толстой (согласно ранее проведенным исследованиям, это наиболее эффективный вариант), и ей нужны крепежные опоры, которые установлены на двух полосах с углублениями для фиксирования средней пластины под определенным углом (рис. 1).

Размеры моделей $S_{1}, S_{2}, S_{3}, X_{1}, X_{3}, X_{4}, X_{5}$ и $\beta$ приведены в таблице. Размеры $X_{2}$ и $X_{6}$ определяются при изготовлении окончательной модели.
При моделировании соударения объектов важную роль играет описание пластических эффектов в материале. Для моделирования мишени и пули использована модифицированная модель Джонсона-Кука как наиболее подходящая к подобным задачам $[9,10]$.

Значения параметров материала стального сплава Armox 560T и пули «АРМ2», относящиеся к модифицированной модели ДжонсонаКука, взяты из публикаций $[8,11]$.

Пуля и область в мишени, подвергаемая наибольшим деформациям, смоделированы с учетом исследований, проведенных по созданию рациональных конечно-элементных моделей (КЭМ) $[10,12]:$ размер конечного элемента задан равным 0,2 мм в области воздействия и 0,3 мм в пуле для получения высокоточных результатов (рис. 2) с помощью программы LS-DYNA [13].

Результаты исследований. Все полученные результаты разделены на три основные части.

1. Угол наклона средней пластины $\beta=35^{\circ}$ и ее толщина $h=3,4,5$, и 6 мм (модели 1-4). У моделей 2-4 суммарная толщина КТП со- 


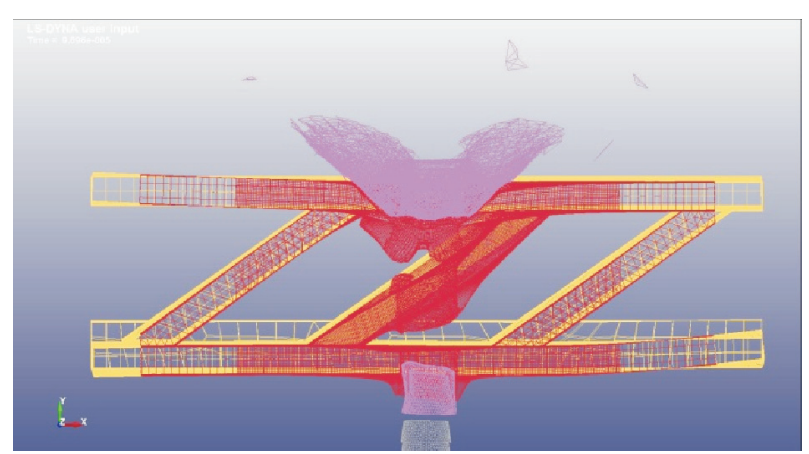

$a$

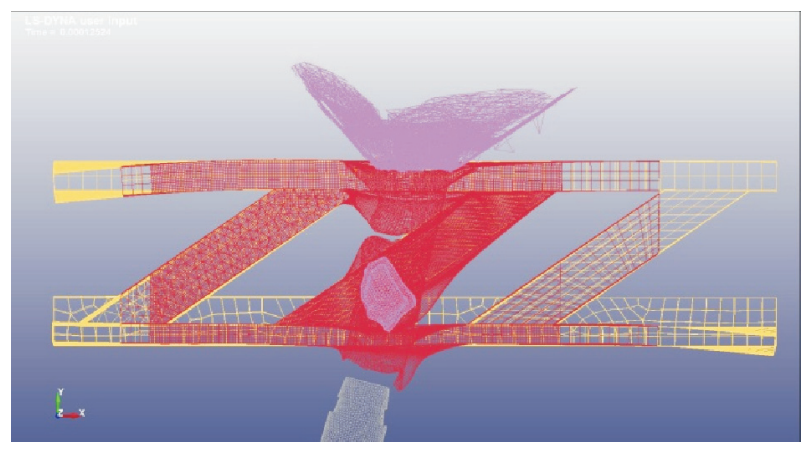

B

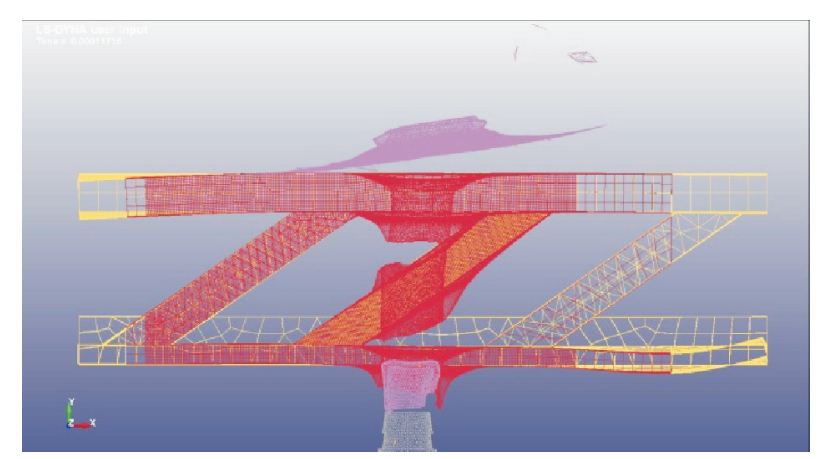

6

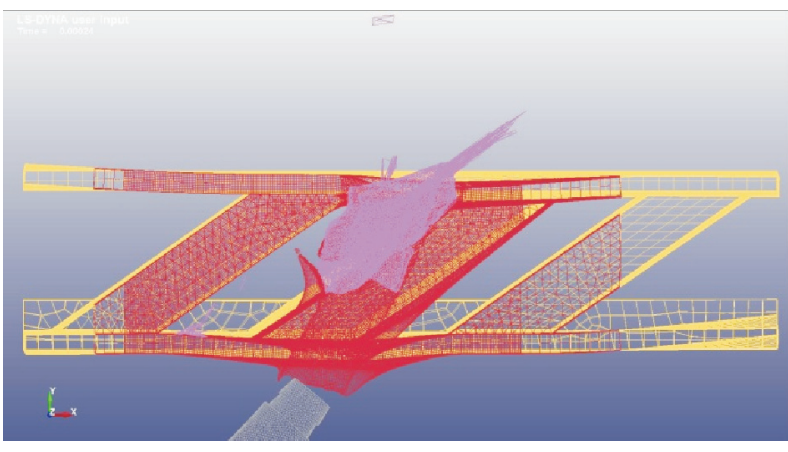

2

Puc. 3. Картины взаимодействия пули «АРМ2» и КТП из сплава Armox 560T для моделей 1 (a), 2 (б), 3 (в) и 4 (2)

ставляет 10 мм, у модели 1-9 мм. На рис. 3 показаны картины взаимодействия пули и КТП из сплава Armox 560T, где видно, что пуля пробила все четыре модели.

2. Угол наклона средней пластины $\beta=30^{\circ}$ или $35^{\circ}$ и ее толщина $h=7$ мм (модели 5-7, см. таблицу). Разница между моделями 5 и 6 состоит в том, что высота модели 6 больше на 4 мм. Как видно из рис. 4, пуля пробила насквозь все пластины модели 5 и осталась в моделях 6 и 7.

3. Угол наклона средней пластины $\beta=30^{\circ}$ и ее толщина $h=8$ мм (модель 8). Верхняя пластина в этой модели, использованная в качестве крепежной, может быть изготовлена из любого легкого материала. В этом случае пуля осталась в пластинах, не пробив их (рис. 5).
На рис. 6 приведены значения остаточных скорости и кинетической энергии пули «АРM2» для всех исследованных моделей. Отметим, что модели 6-8 обеспечивают уровень защиты BR7 по европейской норме EN 1063.

Если сравнить эти модели с пластинами аналогичного размера по массе и результатам (остаточной скорости пули), выявленным в работе [8], то можно получить почти эквивалентную толщину по массе и результатам для каждой модели (рис. 7).

Из рис. 7 видно, что у моделей 1-3 эквивалентная толщина по результатам меньше, чем по массе. В то же время в моделях 4 и 5 эквивалентная толщина по результатам больше, чем по массе, но они не обеспечивают

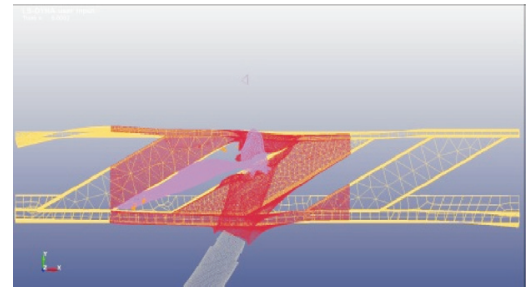

$a$

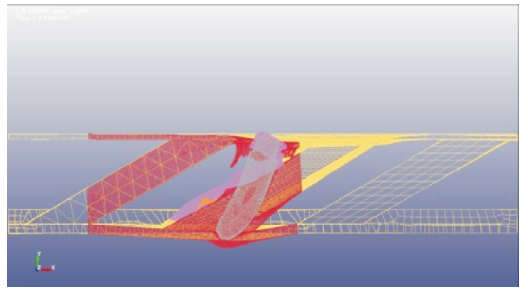

6

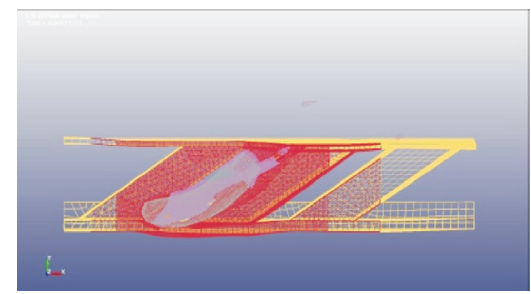

B

Puc. 4. Картины взаимодействия пули «АРМ2» и КТП из сплава Armox 560Т для моделей 5 (a), 6 (б) и 7 (в) 


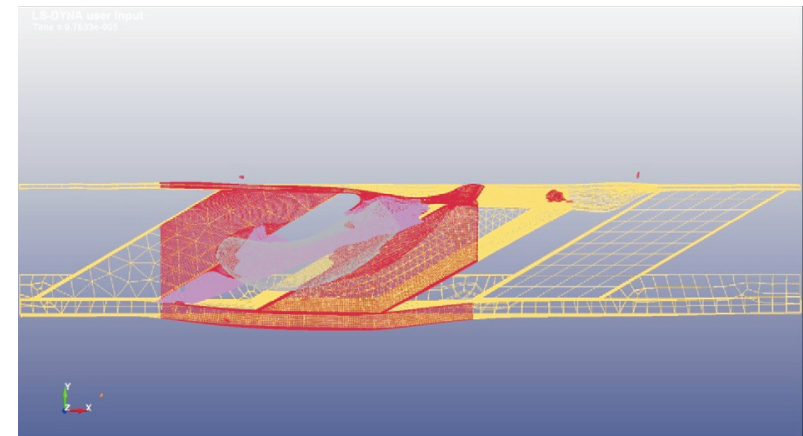

Puc. 5. Картина взаимодействия пули «АРМ2» и КТП из сплава Armox 560Т для модели 8

уровень защиты BR7 по европейской норме $\mathrm{EN}$ 1063. У моделей 6-8 эквивалентная тол-

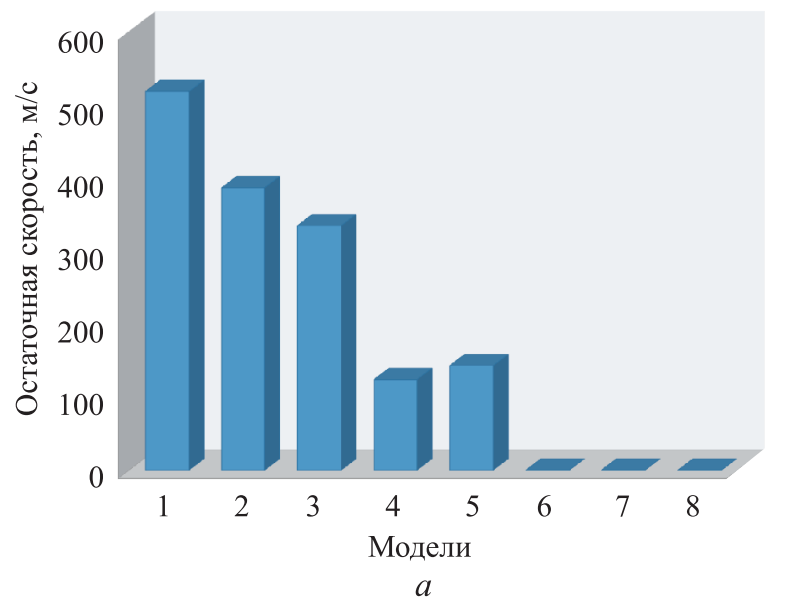

щина по результатам больше, чем по массе, и они соответствуют требуемому уровню защиты.

Отсюда следует, что модель 7 является наилучшей по массе и результатам и дает возможность уменьшить массу примерно на $15 \%$ по сравнению с таковой для одно- и двухслойных конструкций, обеспечивающих тот же уровень защиты. Кроме того, верхнюю пластину можно выполнить из легкого материала, что позволит снизить массу до $7 \%$.

К недостаткам этих моделей относятся сложность изготовления и высокая стоимость. Однако использование пресс-формы для монтажа в случае крупного производства позволит сократить его время и затраты. Применение

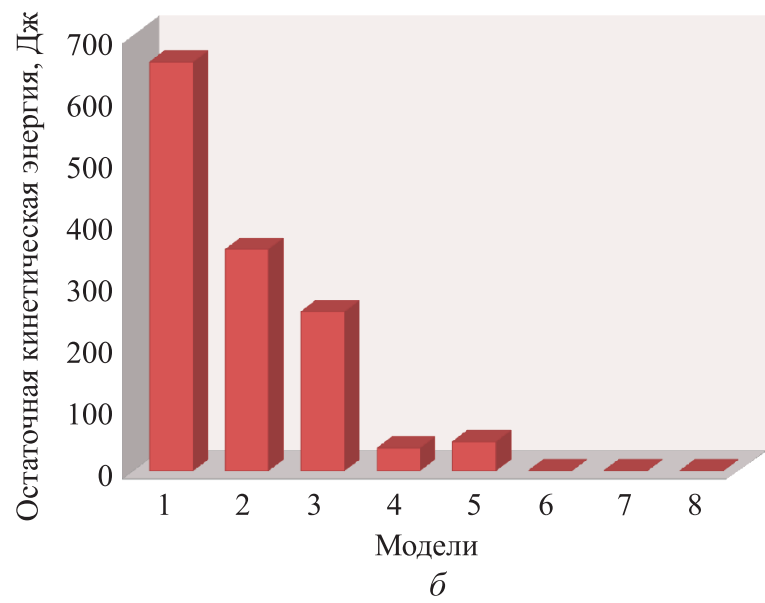

Puc. 6. Значения остаточных скорости (a) и кинетической энергии (б) пули «АРМ2» для исследованных моделей

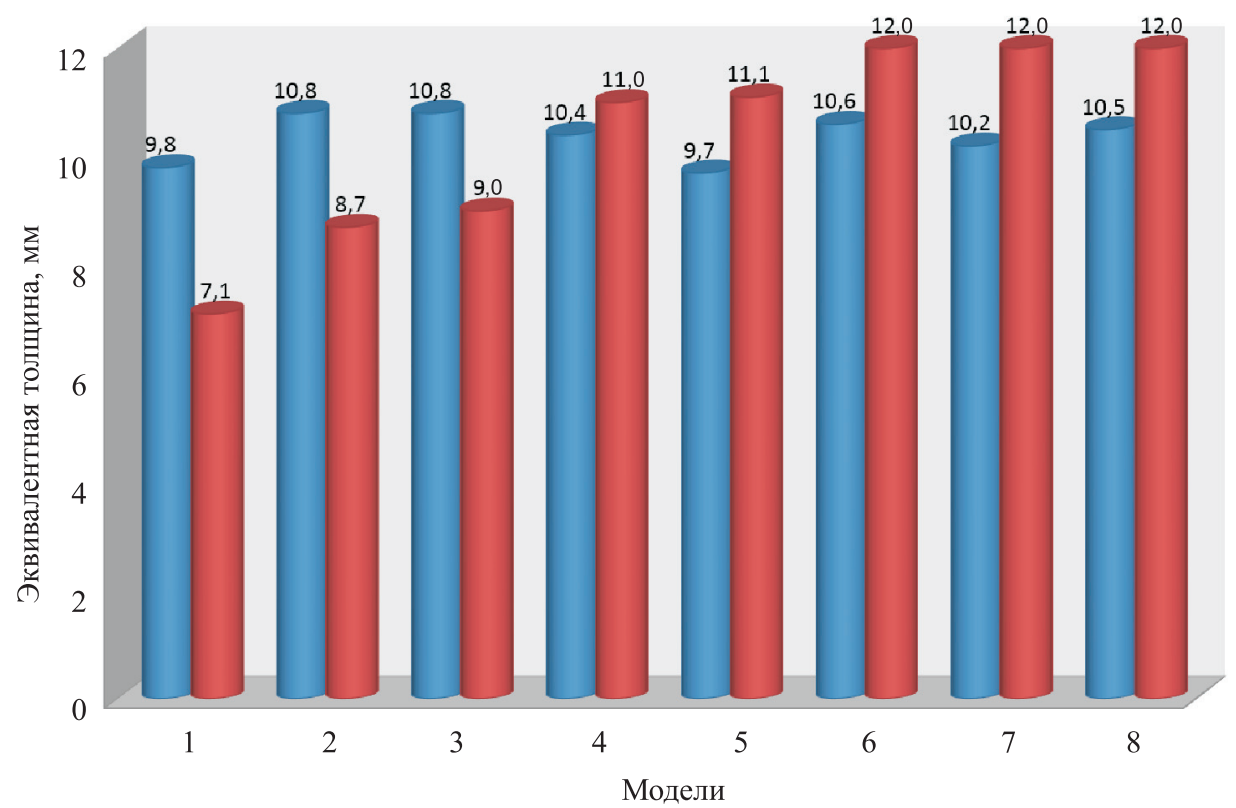

Puc. 7. Значения эквивалентной толщины по массе $(\square)$ и результатам $(\square)$ для исследованных моделей 
обычной стали для крепежных опор полос также снизит стоимость модели.

Возможна дальнейшая разработка КТП для обеспечения защиты против взрыва путем использования поглощающих энергию материалов между пластинами (что предполагает дополнительные исследования).

\section{Выводы}

1. Применение КТП для бронированных кабин обеспечивает требуемую защиту при меньшей массе конструкции (по сравнению с таковой для кабины из одно- или двухслойных бро- непластин) с сохранением внутренних размеров кабины.

2. Модель 7, имеющая наибольшую толщину средней пластины (7 мм), угол наклона $35^{\circ}$ и минимальную толщину наружной (1 мм) и внутренней (2 мм) пластин, является лучшей с точки зрения защиты и массы.

3. Модели 6 и 8 допустимо использовать для обеспечения уровня защиты BR7, а модели 4 и 5 - для уровня защиты меньше, чем BR7.

4. Изготовление средней пластины толщиной до 5 мм является нецелесообразным (с точки зрения защиты и массы, модели 1-3).

\section{Литература}

[1] Teng X., Wierzbicki T., Huang M. Ballistic resistance of double-layered armor plates. International Journal of Impact Engineering, 2008, no. 35, pp. 870-884.

[2] Dey S., Børvik T., Teng X., Wierzbicki T., Hopperstad O.S. On the ballistic resistance of double-layered steel plates: An experimental and numerical investigation. International Journal of Solids and Structures, 2007, no. 44, pp. 6701-6723.

[3] Børvik T., Dey S., Clausen A.H. Perforation resistance of five different high-strength steel plates subjected to small-arms projectiles. International Journal of Impact Engineering, 2009, no. 36, pp. 948-964.

[4] Teng X., Dey S., Børvik T., Wierzbicki T. Protection performance of double-layered metal shields against projectile impact. Journal of Mechanics of Materials and Structures, 2007, vol. 2, pp. 1309-1329.

[5] Flores-Johnson E.A., Saleh M., Edwards L. Ballistic performance of multi-layered metallic plates impacted by a 7.62-mm APM2 projectile. International Journal of Impact Engineering, 2011, no. 38, pp. 1022-1032.

[6] Полунгян А.А., ред. Проектирование полноприводных колесных машин. В 3 m. T. 3. Москва, Изд-во МГТУ им. Н.Э. Баумана, 2008. 432 с.

[7] Chang Qi. A magic cube approach for crashworthiness and blast protection designs of structural and material systems. PhD dissertation, The University of Michigan, 2008. $207 \mathrm{p}$.

[8] Зузов В.Н., Шаш Н. Анализ противопульной стойкости броневых сталей иностранного производства. Наука и образование. Научное издание, 2017, № 5, с. 21-41. URL: http://technomag.edu.ru/jour/article/view/1156 (дата обращения 15 ноября 2017).

[9] Børvik T., Olovsson L., Dey S., Langseth M. Normal and oblique impact of small arms bullets on AA6082-T4 aluminium protective plates. International Journal of Impact Engineering, 2011, vol. 38(7), pp. 577-589.

[10] Шаш Н.С., Зузов В.Н. Влияние параметров алюминиевых сплавов на сопротивление проникания пуль 7.62 «Шар НАТО» и «АРМ2». Наука и образование. Научное издание, 2016, № 11, с. 15-27. URL: http://technomagelpub.elpub.ru/jour/article/view/1075 (дата обращения 15 ноября 2017).

[11] Børvik T., Dey S., Clausen A.H. Perforation resistance of five different high-strength steel plates subjected to small-arms projectiles. International Journal of Impact Engineering, 2009, vol. 36, no. 7, pp. 948-964.

[12] Шаш Н., Зузов В.Н. Численное моделирование проникания пуль стрелкового оружия в пластины из алюминиевых сплавов с использованием модифицированной модели Джонсона-Кука. Наука и образование. Научное издание, 2017, № 1, с. 1-19. URL: http://technomag.edu.ru/jour/article/view/922 (дата обращения 15 ноября 2017).

[13] Hallquist J.O. LS-DYNA Keyword User's Manual. Version 971. California, Livermore Software Technology Corporation, 2007. 


\section{References}

[1] Teng X., Wierzbicki T., Huang M. Ballistic resistance of double-layered armor plates. International Journal of Impact Engineering, 2008, vol. 35, pp. 870-884.

[2] Dey S., Børvik T., Teng X., Wierzbicki T., Hopperstad O.S. On the ballistic resistance of double-layered steel plates: An experimental and numerical investigation. International Journal of Solids and Structures, 2007, vol. 44, pp. 6701-6723.

[3] Børvik T., Dey S., Clausen A.H. Perforation resistance of five different high-strength steel plates subjected to small-arms projectiles. International Journal of Impact Engineering, 2009, vol. 36, pp. 948-964.

[4] Teng X., Dey S., Børvik T., Wierzbicki T. Protection performance of double-layered metal shields against projectile impact. Journal of Mechanics of Materials and Structures, 2007, vol. 2, pp. 1309-1329.

[5] Flores-Johnson E.A., Saleh M., Edwards L. Ballistic performance of multi-layered metallic plates impacted by a 7.62-mm APM2 projectile. International Journal of Impact Engineering, 2011, no. 38, pp. 1022-1032.

[6] Polungian A.A. Proektirovanie polnoprivodnykh kolesnykh mashin [Design of four-wheel drive wheeled vehicles]. In 3 vol. Vol. 3. Moscow, Bauman Press, 2008. 432 p.

[7] Chang Qi. A magic cube approach for crashworthiness and blast protection designs of structural and material systems. PhD dissertation, The University of Michigan, 2008. $207 \mathrm{p}$.

[8] Zuzov V.N., Shash N. Analiz protivopul'noi stoikosti bronevykh stalei inostrannogo proizvodstva [Analysis of Anti-Bullet Resistance of Armored Steels of Foreign Manufacture]. Nauka i obrazovanie. Nauchnoe izdanie [Science and Education]. 2017, no. 5, pp. 21-41. Available at: http://technomag.edu.ru/jour/article/view/1156 (accessed 15 November 2017).

[9] Børvik T., Olovsson L., Dey S., Langseth M. Normal and oblique impact of small arms bullets on AA6082-T4 aluminium protective plates. International Journal of Impact Engineering, 2011, vol. 38(7), pp. 577-589.

[10] Shash N.S., Zuzov V.N. Vliianie parametrov aliuminievykh splavov na soprotivlenie pronikaniia pul' 7.62 «Shar NATO» i «APM2» [Impact of Aluminum Alloy Parameters on Penetration Resistance of the Bullets 7.62 «Ball NATO» and «ARM2»]. Nauka i obrazovanie. Nauchnoe izdanie [Science and Education]. 2016, no. 11, pp. 15-27. Available at: http://technomagelpub.elpub.ru/jour/article/view/1075 (accessed 15 November 2017).

[11] Børvik T., Dey S., Clausen A.H. Perforation resistance of five different high-strength steel plates subjected to small-arms projectiles. International Journal of Impact Engineering, 2009, vol. 36, no. 7, pp. 948-964.

[12] Shash N., Zuzov V.N. Chislennoe modelirovanie pronikaniia pul' strelkovogo oruzhiia v plastiny iz aliuminievykh splavov s ispol'zovaniem modifitsirovannoi modeli DzhonsonaKuka [Modified Johnson-Cook Model-based Numerical Simulation of Small Arms Bullets Penetration in the Aluminum Alloy Plates]. Nauka i obrazovanie. Nauchnoe izdanie [Science and Education]. 2017, no. 1, pp. 1-19. Available at: http://technomag.edu.ru/jour/ article/view/922 (accessed 15 November 2017).

[13] Hallquist J.O. LS-DYNA Keyword User's Manual. Version 971. California, Livermore Software Technology Corporation, 2007.

Статья поступила в редакцию 06.02.2018

\section{Информация об авторах}

ЗУзОВ Валерий Николаевич (Москва) - доктор технических наук, профессор кафедры «Колесные машины». МГТУ им. Н.Э. Баумана (105005, Москва, Российская Федерация, 2-я Бауманская ул., д. 5, стр. 1, е-mail: zuzvalery@rambler.ru).

ШАШ Небрас (Москва) - аспирант кафедры «Колесные машины». МГТУ им. Н.Э. Баумана (105005, Москва, Российская Федерация, 2-я Бауманская ул., д. 5, стр. 1, e-mail: nebrasshash@mail.ru).

\section{Information about the authors}

ZUZOV Valeriy Nikolaevich (Moscow) - Doctor of Science (Eng.), Professor, Department of Wheeled Vehicles. Bauman Moscow State Technical University (105005, Moscow, Russian Federation, $2^{\text {nd }}$ Baumanskaya St., Bldg. 5, Block 1, e-mail: zuzvaleru@rambler.ru).

SHASH Nebras (Moscow) - Postgraduate, Department of Wheeled Vehicles. Bauman Moscow State Technical University (105005, Moscow, Russian Federation, $2^{\text {nd }}$ Baumanskaya St., Bldg. 5, Block 1, e-mail: nebrasshash@mail.ru). 